\title{
Erratum to: Cobalt phosphide nanoarrays with crystalline- amorphous hybrid phase for hydrogen production in universal-pH
}

Hyunseok Yoon ${ }^{\S}$, Hee Jo Song ${ }^{\S}$, Bobae Ju, and Dong-Wan Kim ( $\left.ه\right)$

School of Civil, Environmental and Architectural Engineering, Korea University, Seoul 02841, Republic of Korea

${ }^{\S}$ Hyunseok Yoon and Hee Jo Song contributed equally to this work.

(C) Tsinghua University Press and Springer-Verlag GmbH Germany, part of Springer Nature 2020

Received: 1 April 2020 / Revised: 12 May 2020 / Accepted: 14 May 2020

\section{Erratum to}

Nano Research 2020, 13(9): 2469-2477

https://doi.org/10.1007/s12274-020-2881-y

The contributions of the first two authors were unfortunately misrepresented on the first page and the first page of the ESM.

Instead of

Hyunseok Yoon, Hee Jo Song, Bobae Ju, and Dong-Wan Kim ( $\varangle)$

School of Civil, Environmental and Architectural Engineering, Korea University, Seoul 02841, Republic of Korea

It should read

Hyunseok Yoon ${ }^{\S}$, Hee Jo Song ${ }^{\S}$, Bobae Ju, and Dong-Wan Kim ( $\left.₫\right)$

School of Civil, Environmental and Architectural Engineering, Korea University, Seoul 02841, Republic of Korea

${ }^{\S}$ Hyunseok Yoon and Hee Jo Song contributed equally to this work.

The online version of the original article can be found at

https://doi.org/10.1007/s12274-020-2881-y 